\title{
TRA ORIENTE E OCCIDENTE: NOTE SULLA CIRCOLAZIONE ARTISTICA NEI 'QUARTIERI’ VENEZIANI DEL LEVANTE ATTRAVERSO I DOCUMENTI*
}

\section{LIVIA BEVILACQUA}

UDC: $930.25: 27-525(262.2) " 13 / 14 "$

Original scientific paper

Manuscript received: 03. 11. 2015.

Revised manuscript accepted: 10. 03. 2016.

DOI: 10.1484/J.HAM.5.111333

\author{
L. Bevilacqua \\ Viale Egeo, 147 \\ oo144 Roma, Italia \\ liviabevilacqua@gmail.com
}

In this paper I emphasise the relevance of the archival documents, in order to understand the extent of the circulation of art works in the Venetian ports of the Levant. A few examples are presented, chosen among those, stored in the Archivio di Stato di Venezia, concerning ecclesiastical properties in Constantinople, Raidestos and Negroponte, in which liturgical vessels, textiles and manuscripts are mentioned. Such lists are very concise, but some preliminary remarks can be drawn out of them, about the quality and provenance of the objects used and preserved in the Venetian churches of the Outremer in the late Middle Ages.

Key words: Liturgical Objects, Venice and Byzantium, Venetian Colonies, Archival Documents, Church, Eastern Mediterranean, Constantinople, Raidestos, Negroponte.

Una lunga tradizione erudita, che passa attraverso Charles du Fresne du Cange e Flaminio Corner, ricorda l'episodio registrato da un documento dell'XI secolo, in cui Ordelaffo Falier "per grazia di Dio dux della Venezia e protosevastòs imperiale” dona a Giovanni Gradenigo, patriarca di Grado, la chiesa di Sant'Akindynos, posta nella regia città di Costantinopoli, « quae est antiquitus de jure et possessione nostri palatii, secundum Imperialis crisovoli confirmationem $»^{1}$. E il 1107 , e già da un venticinquennio i Veneziani hanno ottenuto emolumenti, dignità pubbliche e un quartiere, con edifici tra cui la chiesa suddetta, come riferisce il doge, in virtù di una crisobolla firmata dall'imperatore Alessio I Comneno nel maggio del $1082^{2}$. La donazione fatta dal Falier al patriarca include tutte le pertinenze e diritti di proprietà spettanti alla chiesa, sia mobili che immobili, compresi forni, ergasteria e persino i pesi e le misure di riferimento per l'esercizio delle attività commerciali, dunque, vi è incluso tutto ciò che rientra nel consueto formulario giuridico di ogni concessione. Ma in questo caso, e in maniera apparentemente insolita, si sotto- linea anche, sebbene en passant, che la chiesa viene donata " cum toto suo thesauro, et paliis, et libris "³.

Sarebbe interessante scoprire in cosa consistessero "il tesoro, i palli e i libri”, della chiesa di Sant'Akindynos - al di là della generica affermazione che in essi si possa immaginare l'arredo tipico di una chiesa medievale - così come sapere se le chiese latine, e nella fattispecie veneziane, nel levante bizantino, fossero adorne di suppellettile importata dalla madrepatria, o, invece, quanto necessario al culto venisse acquistato o fatto realizzare in situ, adattandosi al gusto e agli usi locali. In definitiva, da un lato in cosa consistesse e quali forme avessero il corredo e l'apparato decorativo di un edificio veneziano (religioso, pubblico, o privato) a Costantinopoli nel Medioevo, e dall'altro chi fosse il responsabile di queste scelte, ovvero quali fossero i committenti o i gestori della circolazione delle opere d'arte nel Mediterraneo. Se infatti, come ha in più occasioni messo in luce Angeliki Laiou, nel pieno e tardo Medioevo, la produzione artistica diventa parte integrante del sistema di transazioni commerciali intrecciato - in particolare - da Venezia, e gli oggetti stessi

\footnotetext{
* Desidero ringraziare il comitato scientifico del $22^{\circ}$ Colloquio Internazionale IRCLAMA, nella persona del Prof. Miljenko Jurković, per avermi offerto la possibilità di presentare in quella sede prestigiosa alcune osservazioni preliminari su questo argomento, oggetto di un assegno di ricerca presso l'Università Ca' Foscari di Venezia e diretto dai Proff. Michela Agazzi e Stefano Riccioni (2014-2015). La mia gratitudine si estende dunque anche a loro e al Prof. Xavier Barral i Altet, per i preziosi consigli in corso d'opera.

${ }^{1} \mathrm{~F}$. CORNER, Ecclesiae Venetae antiquis documentis nunc etiam primus editis illustratae ac in decades distributae, Decas IV-V, Venetiis, 1749, p. 66-71; L. F. TAFEL, G. M. THOMAS (hrsg.), Urkunden zur älteren Handels- und Staatsgeschichte der Republik Venedig mit besonderer Beziehung auf Byzanz und die Levante, I, Wien, 1856, p. 67-74, nr. 32: « [...] nos quidem Ordelafus Faletro gratia Dei Venetie Dux et Imperialis Protosevastos [...] ab hodie in antea damus, et concedimus vobis quidem domno Johanni Gradonico Venerabili Gradensi Patriarchae et vestris successoribus in perpetuum videlicet Ecclesiam Beati Archidani cum omni suo territorio et benefitio positam in regali urbe Constantinopoli quae est antiquitus de jure et possessione nostri palatii, secundum Imperialis crisovoli confirmationem. »

${ }^{2}$ Per il testo completo del documento si rimanda a I trattati con Bisanzio. 992-1198, a cura di M. Pozza, G. Ravegnani, Venezia, 1993 (Pacta Veneta, 4). Il 1082, come sottolinea M. Pozza (Ibid., pp. 35-36), è la datazione più verosimile per la redazione documento, benché siano state proposte in passato date alternative quali il 1084 e il 1092.

3 «Hanc [...] ecclesiam cum toto suo thesauro, et paliis, et libris et cum omnibus suis habentiis et pertinentiis ab intus et foris quas nunc habet et in antea aliquo modo habitura est cum suis argasteriis universis, et cum suo mankipio, et furno, et cum omnibus stateris et rubis, et ponderibus, et cum cunctis metris tam ad oleum quam ad vinum, et cum omnibus nostris tabernis quae in praedicta urbe sub nostra potestate esse videntur [...], ita in vestram, et Gradensis Ecclesiae et successorum vestrorum damus, atque in perpetuum concedimus plenissimam potestatem habendi, tenendi, meliorandi, edificandi, fruendi, ordinandi, disponendi, inquirendi et in perpetuum possidendi. », cfr. F. CORNER, op. cit. (n. 1), e L. F. TAFEL, G. M. THOMAS (hrsg.), op. cit. (n. 1), p. 68.
} 
divengono una sorta di "moneta di scambio", occorrerà forse verificare più attentamente quanto la stessa studiosa asseriva circa il fatto che, nel XIII secolo, « The substitution of the Venetian for the Byzantine dominance in the Byzantine Empire is paralleled by the substitution of Venetian-made objects for Byzantine ones $»^{4}$.

Si tratta di un tema di ricerca ancora poco indagato, se non limitatamente ad alcune aree geografiche e ad alcuni contesti meglio documentati. Nell'ambito di un progetto di ricerca più ampio che ho avviato, mi pongo l'obiettivo di delineare le caratteristiche della produzione artistica in senso lato, le modalità della circolazione di opere d'arte e di artisti, le problematiche legate alla committenza e al commercio di beni di lusso, nei quartieri veneziani delle città bizantine del Mediterraneo, a partire da quello di più antica formazione, situato a Costantinopoli. L'ambito cronologico interessato da questo lavoro è assai ampio, giacché si prende in considerazione l'intero periodo che va dalle prime attestazioni della presenza veneziana a Costantinopoli - dunque all'incirca il IX secolo - fino alla conquista ottomana della città, ma in questa occasione mi concentrerò soprattutto sul periodo successivo alla Quarta Crociata e all'occupazione latina. Si tratta di un problema delicato e dalle molteplici implicazioni, per affrontare il quale è necessario percorrere parallelamente diverse strade, incrociando i dati in maniera certosina: dalla ricerca documentaria, allo studio analitico di singole opere e personaggi identificabili come committenti, produttori o intermediari, all'analisi comparativa di eventuali opere d'arte superstiti. In queste pagine desidero dar conto di alcuni risultati della prima fase della ricerca, in cui ho percorso la via dello spoglio dei documenti d'archivio, primo passo necessario per offrire qualche puntello ad ogni riflessione successiva. Tale lavoro è complicato dalla perdita dei materiali e della discontinuità di quelli che sono giunti fino a noi. Tuttavia, attraverso una ricerca a raggio molto ampio, è possibile ottenere qualche risposta che può rivelarsi di grande interesse. Tra i documenti da cui si può trarre qualche indizio in questa direzione, quelli a cui farò riferimento ci condurranno in un itinerario a ritroso, lungo le rotte mediterranee del Medioevo, e ci faranno sostare in alcuni porti veneziani, da Costantinopoli alla laguna.

\section{COSTANTINOPOLI}

Se le attività marittime nella capitale bizantina erano state, inizialmente, equamente distribuite lungo tutte le

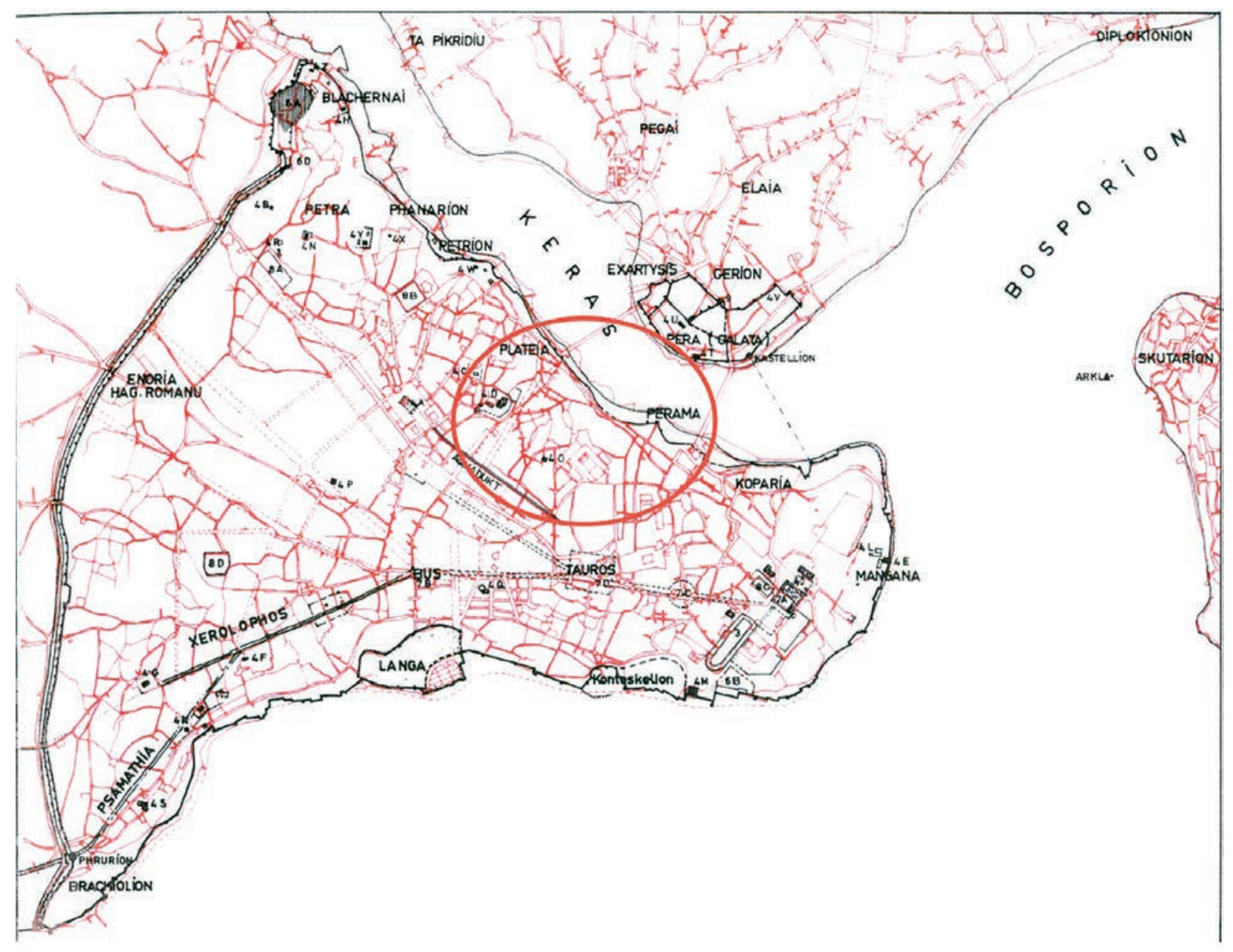

Fig. 1: Mappa della città di Costantinopoli con indicazione dell'ubicazione del quartiere veneziano (elaborazione da Müller-Wiener 1977).

${ }^{4}$ A. E. LAIOU, Venice as a Centre of Trade and of Artistic Production in the Thirteenth Century, in H. Belting (ed.), Il Medio Oriente e l'Occidente nell'arte del XIII secolo, Bologna, 1982, p. 11-26 (rist. in EAD., Byzantium and the Other: Relations and Exchanges, C. Morrisson, R. Dorin [ed.], Farnham, 2012, nr. IX). ${ }^{5}$ Su questo problema si veda P. MAGDALINO, The Maritime Neighborhoods of Constantinople: Commercial and Residential Functions, Sixth to Twelfth Centuries, in Dumbarton Oaks Papers, 54, 2000, p. 209-226 (rist. in ID., Studies on the History and Topography of Byzantine Constantinople, Aldershot, 2007, n. III). 
insenature della costa che circonda la penisola su cui sorge Costantinopoli, la maggior concentrazione di tali attività si verrà pian piano fissando, prevalentemente, nella zona sud, affacciata sul Mar di Marmara5. Invece, a nord della stessa penisola, lungo la sponda meridionale del Corno d'Oro, si stabiliranno le comunità occidentali, installandovi le basi per le proprie attività commerciali e diplomatiche in città, ma anche - in un periodo più tardo - in funzione di scalo per le rotte che conducevano, più a Oriente, verso l'Asia centrale e orientale. Quest'area (fig. 1), pur non direttamente affacciata sul Mar Mediterraneo, ne diventa così un punto di snodo cruciale, un vero porto naturale strategicamente situato tra il Mar di Marmara (e dunque, oltre i Dardanelli, il Mediterraneo) e il Mar Nero. Brulicante di attività mercantili, marittime, ma anche in larga parte agricole, il settore nord della città doveva così mostrarsi - mutatis mutandis soprattutto in termini di densità abitativa - non del tutto dissimile, in spirito, a quello che è, nella moderna Istanbul, l'animato quartiere commerciale di Eminönü.

Il documento citato all'inizio di questo contributo, che registra la donazione della chiesa di Sant'Akindynos, è ben noto agli studiosi che si sono occupati di disegnare la fisionomia del "quartiere" veneziano di Costantinopoli, in età comnena e paleologa (mi riferisco in particolare a Ennio Concina, Chryssa Maltezou, Aygül Ağır, David Jacoby, per nominarne solo alcuni) ${ }^{6}$. Grazie a questo documento e a molti altri, ma anche grazie ad alcuni indizi ricavabili dalla sistemazione di questa parte della città in epoca ottomana - e nonostante l'assenza di scavi archeologici, in un'area oggi intensamente edificata - è possibile farsi un'idea della topografia del luogo nelle sue linee generali. I suoi confini erano indicati in effetti anche nella crisobolla di Alessio Comneno del 1082 che, peraltro, si limitava forse a normalizzare una situazione di fatto. L'imperatore aveva concesso a Venezia alcuni stabili situati nella zona dell'embolus, cioè del portico, di Perama, dalla porta Ebraica alla porta di Vigla, magazzini con i locali ad essi soprastanti e che si aprivano sul portico, e tre scalae, o approdi, situati nella medesima zona, fuori dalle mura marittime 7 . Con quell'editto il doge era stato inoltre nominato protosevastòs (cioè "primo augusto") in perpetuo (un titolo onorifico quindi che non veniva legato alla persona allora in carica) mentre al patriarca veniva concesso l'attributo di ypèrtimos, cioè "onorabilissimo"; in aggiunta - sempre stando alla crisobolla - la chiesa di San Marco a Venezia avrebbe dovuto ricevere annualmente un tributo da ogni amalfitano proprietario di una bottega a Costantinopoli o in altri territori dell'impero, decretando il netto vantaggio dei veneziani sulle altre comunità occidentali che avevano interessi cospicui sul Bosforo ${ }^{8}$. I privilegi commerciali che accompagnavano queste concessioni erano senza precedenti, e ciò che si richiedeva a Venezia in cambio era di fornire appoggio e supporto logistico all'imperatore bizantino in caso di guerra. E importante ricordare il contenuto dell'editto ora citato, perché esso (nonostante un atteggiamento, da parte delle autorità locali, assai altalenante nei confronti dei veneziani) verrà ribadito anche nei successivi documenti che regoleranno i rapporti tra i veneziani del Levante e l'impero bizantino, praticamente fino alla scomparsa stessa di quest'ultimo. Anzi, il rinnovo dei privilegi economici di Venezia nel territorio imperiale diverrà uno strumento per assicurarsi il sostegno della Serenissima nei momenti di maggiore difficoltà per l'impero'.

A dispetto delle (come detto) scarsissime testimonianze archeologiche, a far riscontro alle fonti documentarie testé citate esiste qualche rara testimonianza grafica antica della localizzazione di almeno uno degli edifici appartenenti a Venezia sul Corno d'Oro. Infatti, in quasi tutte le versioni più antiche della mappa di Costantinopoli che accompagna il Liber insularum archipelagi di Cristoforo Buondelmonti, opera composta nel XV secolo e pervenutaci in numerose versioni manoscritte illustrate, nella zona in cui doveva trovarsi il quartiere veneziano, è indicata la presenza di un edificio di forma basilicale con campanile, che doveva spiccare tra gli altri per le sue dimensioni. Esso rimane anonimo nella maggior parte degli esemplari giunti fino a noi, ma in almeno due di essi, ovvero nel ms. CM 289 della Biblioteca Civica di Padova (fig. 2) e nel più tardo ms. 15 del fondo Donà dalle Rose della Biblioteca del Museo Correr di Venezia (fig. 3), esso è accompagnato dalla didascalia "Sanctus Marcus". Non vi è dubbio, come è stato ricordato anche da Claudia

\footnotetext{
${ }^{6}$ Oltre alla panoramica offerta da P. MAGDALINO, Constantinople médiévale. Etudes sur l'évolution des structures urbaines, Paris, 1996, p. 78-90, e, sui quartieri marittimi della capitale, ID., op. cit. (n. 5), si vedano, per un approccio più specifico: C. MALTEZOU, Il quartiere veneziano di Costantinopoli (Scali marittimi), in Thesaurismata, 15, 1978, pp. 30-61; A. BERGER, Zur Topographie der Ufergegend am Goldenen Horn in der byzantinischen Zeit, in Istanbuler Mitteilungen, 45, 1995, p. 149-165; D. JACOBY, The Venetian Quarter of Constantinople from 1082 to 1261: Topographical Considerations, in C. Sode, S. Takács (eds.), Novum Millennium. Studies on Byzantine History and Culture dedicated to Paul Speck, Aldershot, 2001, pp. 154-159 (rist. in ID., Commercial Exchange across the Mediterranean: Byzantium, the Crusader Levant, Egypt and Italy, Aldershot, 2005, n. III); E. CONCINA, Il quartiere veneziano di Costantinopoli, in G. Benzoni (ed.), L'eredità greca e l'ellenismo veneziano, Firenze, 2002, p. 157-170; D. JACOBY, Houses and Urban Layout in the Venetian Quarter of Constantinople: Twelfth and Thirteenth Centuries, in K. Belke, E. Kislinger (eds.), Byzantina Mediterranea. Festschrift für Johannes Koder zum 65. Geburtstag, Wien 2007, pp. 269-282; A. AĞIR, İstanbul'un Eski Venedik Yerleșimi ve Dönüșümü, Istanbul, 2013.

7 «Ad haec donat eis et ergasteria que sunt in embolo Peramatis cum solariis suis, que habent introitum et exitum in embolum, que procedunt ab Ebraica usque ad Viglan, queque habitantur et que non habitantur, et in quibus Venetici permanent et Greci sicut ergasteriis, et maritimas III scalas que in predicto spatio terminantur. » in M. POZZA, G. RAVEGNANI (eds.), I trattati con Bisanzio. 992-1198, Venezia, 1993 (Pacta Veneta, 4).

${ }^{8}$ Sulle attività commerciali degli amalfitani a Bisanzio e nell'oriente mediterraneo si vedano almeno: V. VON FALKENHAUSEN, Il commercio di Amalfi con Costantinopoli e il Levante nel secolo XII, in O. Banti (ed.), Amalfi Genova Pisa e Venezia. Il commercio con Costantinopoli e il vicino Oriente nel secolo XII. Atti della Giornata di Studio (Pisa, 27 maggio 1995), Ospedaletto 1998, p. 19-38; D. JACOBY, Commercio e navigazione degli Amalfitani nel Mediterraneo orientale: sviluppo e declino, in B. Figliuolo, P. F. Simbula (eds.), Interscambi socio-culturali ed economici fra le città marinare d'Italia e l'Occidente dagli osservatorî mediterranei. Atti del Convegno Internazionale di Studi in memoria di Ezio Falcone (1938-2011), Amalfi 14-16 maggio 2011, Amalfi 2014 , p. 89-128. 9 Per una sintesi delle vicende che accompagnano la promulgazione della prima crisobolla e il susseguirsi di patti che regolano la presenza veneziana nei territori bizantini si veda, per il periodo compreso tra XI e XII secolo, G. RAVEGNANI, Il commercio veneziano nell'impero bizantino, in O. Banti (ed.), Amalfi Genova Pisa e Venezia. Il commercio con Costantinopoli e il vicino Oriente nel secolo XII. Atti della Giornata di Studio (Pisa, 27 maggio 1995), Ospedaletto 1998, p. 55-74. Per i testi dei documenti, nonché per il periodo successivo: M. POZZA, G. RAVEGNANI (eds.), op. cit. (n. 7); IID. (eds.), I trattati con Bisanzio. 1265-1285, Venezia, 1996 (Pacta Veneta, 6). In generale, rimangono punti di riferimento: D. M. NICOL, Venezia e Bisanzio, Milano, 199o (I ed. Cambridge, 1988); G. RAVEGNANI, Bisanzio e Venezia, Bologna 2006.
} 


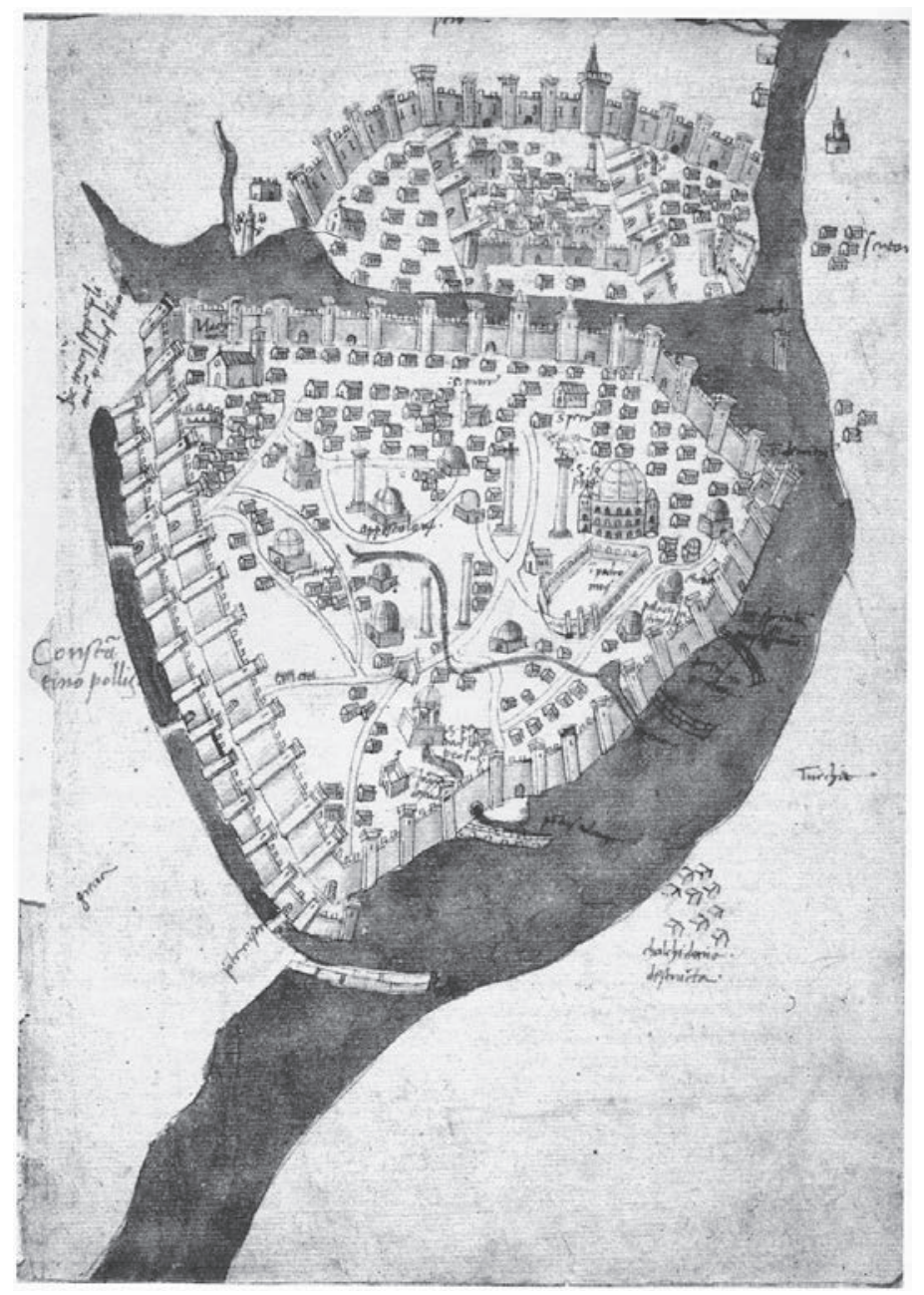

Fig. 2: Padova, Biblioteca Civica, ms. CM 289, f. 18r, Cristoforo Buondelmonti, Liber insularum Archipelagi, mappa della città di Costantinopoli (da Barsanti 2001).

Barsanti ${ }^{10}$, che si tratti di un'altra delle chiese di cui siamo a conoscenza che, nel quartiere costantinopolitano della Serenissima, era officiata dal clero veneziano: San Marco de embulo venetorum. Oltre a quella già citata di Sant'Akindynos, vi erano anche altre due chiese che non hanno lasciato alcuna traccia visiva: Santa Maria e San Nicola de embulo, che saranno invece spesso menzionate dai documenti in seguito.

Se la vocazione commerciale della comunità che oggi chiameremmo "levantina" si rifletteva anche nella funzione di tali istituti di essere il ricovero dei pesi e delle misure necessarie per svolgere tale attività, dal punto di vista giuridico e amministrativo le chiese veneziane di Costantinopoli (e di tutto il Levante) ricadevano sotto la giurisdizione di chiese e monasteri della laguna, e pertanto la lettura dei documenti raccolti nei fondi archivistici relativi a questi ultimi appare indispensabile, dal momento che, sul versante greco, nulla o quasi si è conservato quanto a materiale

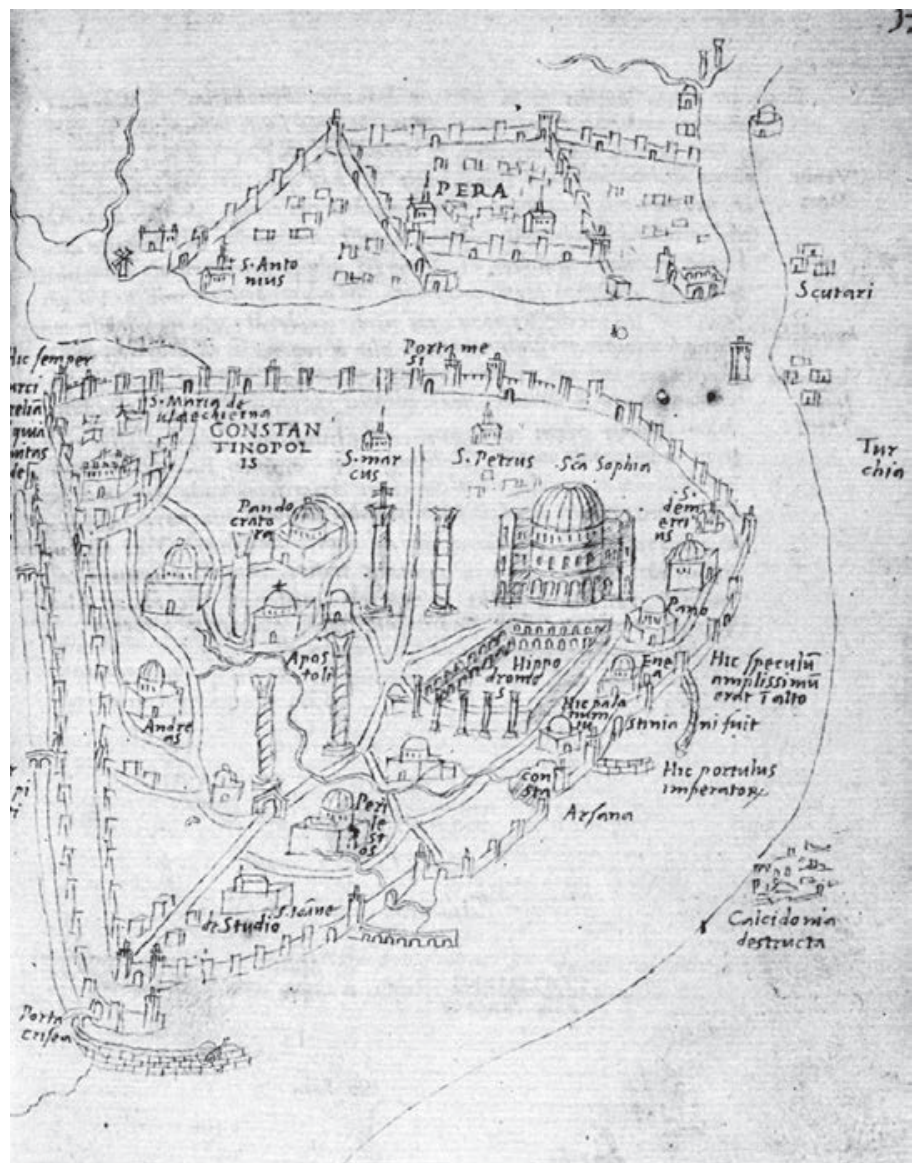

Fig. 3: Venezia, Biblioteca del Museo Correr, ms. 15 Fondo Donà dalle Rose, f. 37r, Cristoforo Buondelmonti, Liber insularum Archipelagi, mappa della città di Costantinopoli (da Barsanti 2001).

d'archivio, mentre tutto ciò che riguardava le dipendenze d'Oltremare delle chiese veneziane, quanto ad amministrazione religiosa, questioni legali, contatti del clero con la casa madre, da un lato, con la popolazione locale, dall'altro, è registrato scrupolosamente in quei faldoni. Il fondo "San Giorgio Maggiore" dell'Archivio di Stato di Venezia è il più ricco in tal senso, poiché questo importante monastero veneziano godeva di un sistema di proprietà oltremare assai capillare. Per quanto concerne la capitale, oltre alla già citata Sant'Akindynos, dipendeva dall'abate di San Giorgio anche la chiesa di San Marco, nonché un considerevole numero di immobili e vasti appezzamenti di terra che, nel XIII secolo, ricoprivano ormai un'area che si estendeva fino alla zona delle Blacherne ${ }^{11}$. Più complicata da ricostruire, a livello documentario, è la vicenda che riguarda la chiesa di Santa Maria, la quale dipendeva dal monastero dei Santi Felice e Fortunato di Ammiana ${ }^{12}$ : quest'ultimo, soppresso nel 1472, è oggi scomparso e il suo archivio, in parte andato disperso, è confluito nel vasto fondo dei "Procuratori di San Marco de Supra". La chiesa di San Nicola, invece, era

\footnotetext{
${ }^{10}$ C. BARSANTI, Costantinopoli e l'Egeo nei primi decenni del XV secolo: la testimonianza di Cristoforo Buondelmonti, in Rivista dell'Istituto Nazionale d'Archeologia e Storia dell'Arte, 56 (III serie, 24), 2001, p. 83-254. A tale articolo si rimanda per un'analisi approfondita delle illustrazioni dei manoscritti di Buondelmonti, relative a Costantinopoli e alle isole egee.

"Come fanno pensare, ad esempio, le indicazioni topografiche presenti nella concessione per lo sfruttamento delle acque fatta dal doge Pietro Ziani all'abate di S. Giorgio Maggiore, datato 1207 (Archivio di Stato di Venezia [ASVe], San Giorgio Maggiore, b. 126 [già 121]), vd. L. F. TAFEL, G. M. THOMAS (hrsg.), op. cit. (n. 1), p. 47-49. Questo documento si riferisce al periodo della dominazione latina della città, ma anche dopo la riconquista bizantina non si constata una riduzione significativa nell'estensione delle proprietà dei veneziani.

${ }^{12}$ L. B. ROBBERT, Rialto Businessmen and Constantinople, 1204-1261, in Symposium on Byzantium and the Italians, $13^{\text {th }}-15^{\text {th }}$ Centuries, Dumbarton Oaks Papers, 49, 1995, p. 43-58, in part. p. 49; E. ORLANDO, "Ad profectum patriae". La proprietà ecclesiastica veneziana in Romània dopo la IV Crociata, Roma, 2005, p. 31-32 e passim.
} 
legata all'omonima chiesa del Lido $^{13}$, e per recuperarne le tracce occorre scandagliare l'omonimo fondo dell'Archivio di Stato di Venezia.

Affittanze e documenti giuridici di vario tipo, ufficiali e pubblici, sono certo assai utili per tentare una ricostruzione ideale dello spazio del quartiere, ma le informazioni sulla circolazione artistica che vi si svolgeva sono assai scarse. È pertanto necessario guardare "oltre" i confini del quartiere veneziano di Costantinopoli: quest'ultimo è infatti un contesto particolarissimo, per via della compresenza fianco a fianco, in uno stesso settore della città, di comunità di varia origine. Spunti alternativi, per comprendere di riflesso il contesto della capitale, arrivano però anche da luoghi differenti. Perciò sarà importante osservare le realtà dei quartieri veneziani nelle altre città portuali bizantine, dove questi gestivano i loro scali in rapporto con le autorità locali, oppure che giunsero a governare autonomamente.

Ciò è peraltro inevitabile, perché, anche volendo focalizzare l'attenzione sul quartiere costantinopolitano, ci si imbatterà in innumerevoli notizie, riferimenti diretti e indiretti, informazioni riguardanti anche le altre città, isole, semplici approdi che, facendo capo alla Serenissima, costellavano il Mediterraneo medievale, rendendolo una sorta di grande mercato, il punto d'incontro dei membri di una comunità, e certamente luogo di scambio culturale, forse molto più di quanto non lo sia ai nostri tempi.

Si presenteranno dunque, qui di seguito, alcuni documenti reperibili nell'Archivio di Stato, che concernono edifici di culto oltremare. Così facendo, ci si allontanerà geograficamente da Costantinopoli, ma sarà interessante poter seguire il filo rosso che, dalla capitale bizantina, ci riporterà in laguna. Si tratta di carte che, ad una lettura attenta, rivelano dati assai interessanti circa gli oggetti che circolavano in siffatti contesti, e permetteranno di immaginarne con più chiarezza l'arredo.

\section{RODOSTO}

Nel fondo "San Nicolò di Lido", tra pergamene ormai quasi polverizzate, si conserva un documento rogato a $\mathrm{Co}$ stantinopoli nel $1231^{14}$. Esso ci informa che Giovanni, vescovo di Rodosto, che aveva precedentemente ricevuto da Stefano, già priore di San Nicola de embulo, 95 iperperi, si è servito di tali denari per acquistare della nuova suppellettile per la propria chiesa; ne promette ora la restituzione ad Alberto, attuale priore di San Nicola, spiegando come essi siano stati adeguatamente spesi: «de quibus yperperis comparavimus pro utilitate et necessitate nostre capelle bacilia duo argenti, anpolletas duas argenti, et terribullum unum argenti adque examita duo, de quibus examitis fecimus duo indumenta id est dalmaticam unam et strictam unam $»^{15}$. Di argenterie per l'uso liturgico ${ }^{16}$, dunque, la chiesa episcopale di Rodosto era carente (anche se non dovevano mancare almeno gli essenziali calice e patena), e il suo corredo è stato così integrato con due bacili per le abluzioni, due ampolle (una per l'acqua e una per il vino), un turibolo per l'incenso; inoltre ci si è procurati due sciamiti, che sono serviti per confezionare una dalmatica e una "tunica stretta".

Purtroppo il documento è estremamente sintetico nel suo elenco, e del tutto silente circa la qualità più propriamente artistica degli oggetti nominati. Ma ciò che è senz'altro interessante è l'attestazione che questi oggetti siano stati "acquistati" in blocco, già pronti, e non fatti eseguire ad hoc da qualche artista incaricato dell'impresa. Solo a proposito dei tessuti si specifica che essi sono serviti a realizzare due vesti liturgiche. Non si esplicita chi le abbia cucite, ma si potrebbe pensare, in questo caso, che si sia trattato di sarti alle dipendenze della chiesa. Basandosi sulla tradizionale attenzione dei veneziani alla scelta dei tessuti serici di produzione bizantina, che poi importavano in Occidente, si potrà forse supporre che, almeno per quanto riguarda questi manufatti (gli sciamiti), il nostro Giovanni abbia condotto il suo "shopping" presso botteghe di Costantinopoli.

Se poi, per quanto riguarda i lavori in argento, si trattasse di laboratori di artigiani bizantini o occidentali è ovviamente impossibile dire. Siamo negli anni della dominazione latina, ed è dunque possibile (sebbene non indispensabile) che, in questo caso, le botteghe responsabili della produzione di tali oggetti fossero "latine". Tuttavia, la natura delle botteghe artigiane a Bisanzio è questione di non facile inquadramento, non solo per la scarsità di informazioni possedute, ma soprattutto per il loro carattere, per così dire, "misto". In esse infatti lavoravano fianco a fianco artisti e artigiani di varia provenienza e background etnico, e vien fatto di domandarsi se non fosse la nazionalità greca o occidentale dei loro proprietari o gestori a farne degli stabilimenti, rispettivamente, bizantini o latini ${ }^{17}$. Sarei tuttavia propensa ad escludere, in questo caso, che il nostro abbia acquistato

\footnotetext{
${ }_{13}$ Ibid. Per alcune indicazioni di carattere storico-archeologico sul monastero di San Nicolò di Lido, si veda L. FABBIANI, La Fondazione monastica di San Nicolò di Lido (1053-1628), Venezia, s.a.

${ }^{14} \mathrm{Il}$ testo è pubblicato in R. MOROZZO DELLA ROCCA, A. LOMBARDO (ed.), Documenti del commercio veneziano nei secoli XI-XIII, Torino 1940, 2, pp. 195-196, nr. 658; sull'episodio qui ricordato si veda anche L. B. ROBBERT, op. cit. (n. 12), p. 54; E. ORLANDO, op. cit. (n. 12), p. 147 , nr. 45.

${ }^{15}$ ASVe, S. Nicolò di Lido, Pergamene, b. 2: « [...] manifestum facimus Nos Iohannes Dei gratia Rodostensis episcopus cum nostris successoribus, quia recepimus a Reverendo dompno Stephano qui fuit prior ecclesie Sancti Nicolay embuli Venetorum Constantinopoli, antecessore de te reverendo patre domino Alberto modo priore suprascripte ecclesie Sancti Nicolay embuli Venetorum, idest yperpera auri bene pensantia Nonaginta quinque, de quibus yperperis comparavimus pro utilitate et necessitate nostre capelle bacilia duo argenti, anpolletas duas argenti, et terribullum unum argenti adque examita duo, de quibus examitis fecimus duo indumenta id est dalmaticam unam et strictam unam, que omnia dicta yperpera fuerunt de perperis bonis suprascripte ecclesie Sancti Nicolay, et que mihi mutuavit in meo periculo. Igitur manifestum facimus quia nos per nos vel per nostrum missum dabimus vel dari faciemus vobis domino Alberto suprascripto priori vel vestro misso aut qui per tempora ibidem prior in eadem ecclesia Sancti Nicolay totam et integram medietatem omnium suprascriptorum yperperorum [...]», 1231, dicembre, V indizione, Costantinopoli.

${ }^{16}$ M. COLLARETA, Arredi, suppellettili, decorazioni mobili, in E. Castelnuovo, G. Sergi (eds.), Arti e storia nel Medioevo. II. Del costruire: tecniche, artisti, artigiani, committenti, Torino, 2003, p. 303-328, in part. p. 313-315, sulla distinzione funzionale e le sue ricadute tecniche, e P. J. GEARY, Oggetti liturgici e tesori della Chiesa, in E. Castelnuovo, G. Sergi (eds.), Arti e storia nel Medioevo. III. Del vedere: pubblici, forme e funzioni, Torino, 2004, p. 275-29o. Benché limitato al periodo romanico A. LEGNER (hrsg.), Ornamenta Ecclesiae. Kunst und Künstler der Romanik. Katalog zur Ausstellung des Schnütgen-Museums in der Josef-Haubrich-Kunsthalle, Köln 1985, Köln 1985, volumi 1 e 3 rispettivamente per il medioevo occidentale e bizantino.

${ }^{17}$ M. BACCI, Greek Painters, working for Latin and non-orthodox patrons in the Late Medieval Mediterranean. Some preliminary remarks, in J. Anderson (ed.), Crossing Cultures: Conflict, Migration and Convergence. The Proceedings of the 32nd International Congress in the History of Art, The University of Melbourne, 13-18 January 2008, Melbourne, 2009, p. 164-168.
} 
uno stock di oggetti importati dalla madrepatria, poiché un significativo incremento dell'esportazione di manufatti di pregio da Venezia verso Bisanzio risulterà evidente solo più tardi, a partire dalla seconda metà del XIV secolo, quando la produzione locale bizantina subirà una flessione a causa della crisi politica ed economica ${ }^{18}$. Come ha sottolineato Anna Ballian, in questo periodo più tardo troveremo persino attestazioni del fatto che gli oggetti liturgici (anche quelli utilizzati nelle chiese greche) erano ormai prodotti in larga misura nelle botteghe di argentieri presenti nelle colonie veneziane: dovevano esisterne a Costantinopoli, e sono documentate a Scutari/Üsküdar, Ragusa/Dubrovnike Candia, la quale ultima fu peraltro la città di nascita di vari orafi cretesi registrati negli archivi di Ragusa negli anni '6o del XIV secolo ${ }^{19}$.

Tornando al nostro acquisto di Rodosto del 1231, dobbiamo comunque supporre che esso sia avvenuto a Costantinopoli, possibilità avvalorata dalla vicinanza di Rodosto (Raidestos, odierna Tekirdağ) ${ }^{20}$, cittadina tracia affacciata sul Mar di Marmara, proprio alla capitale bizantina, vicinissima dal punto di vista geografico nonché centro artistico fiorente. Ma stiamo entrando nell'ambito della speculazione, ed è bene fermarci qui. Infatti, purtroppo sappiamo ancora davvero poco delle manifatture di lusso costantinopolitane, soprattutto di quelle attive negli ultimi due secoli della vita di Bisanzio, anche se le ricerche più recenti sembrano indirizzate allo studio più circostanziato di questo tema ${ }^{21}$; e ancora molto lavoro è da fare anche su questo cruciale problema nell'epoca della dominazione latina.

\section{NEGROPONTE}

L'isola di Eubea/Negroponte, è nodo di transito e di scambio di importanza capitale per il commercio di Venezia nel Mediterraneo già durante il regno dei Comneni ${ }^{22}$. Dopo la Quarta Crociata e la successiva spartizione dei territori conquistati dai latini, una grossa fetta dell'isola diviene a tutti gli effetti dominio veneziano. A tale evento si accompagnano interventi edilizi e urbanistici, in primis la fortificazione della città, che occupava una posizione chiave sullo stretto dell'Euripo. Di quest'opera di fortificazione e del notevole sviluppo del tessuto urbano al suo interno, ci rimane forse

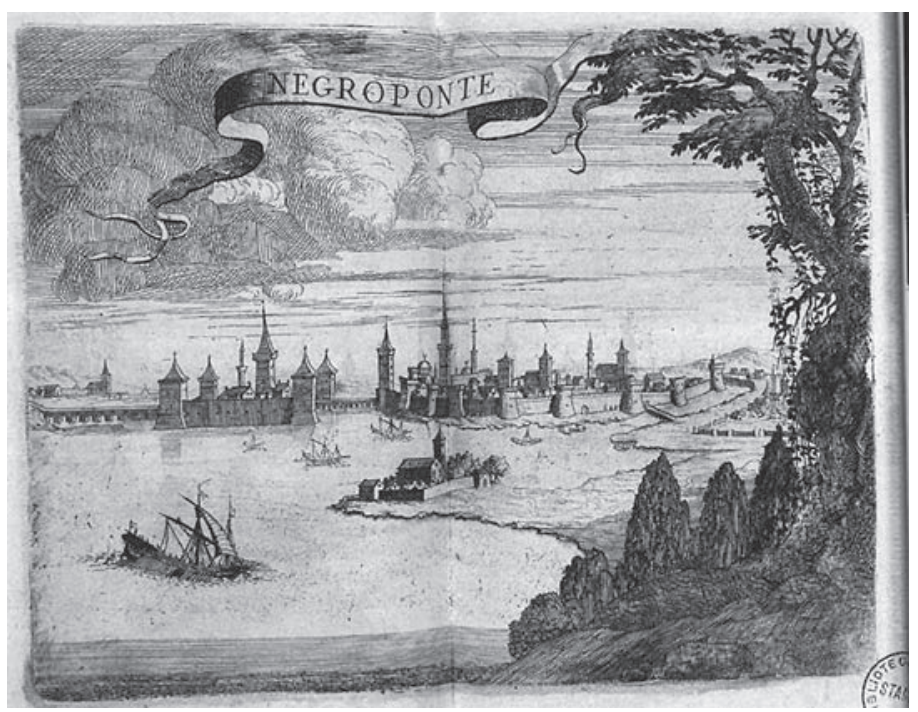

Fig. 4: Venezia, Biblioteca Nazionale Marciana, 16.d.288, V.M. Coronelli, Memorie istoriografiche della Morea e Negroponte, Venezia, 1686, tav.

39 (da http://www.internetculturale.it/jmms/iccuviewer/iccu.jsp?id=mag GEO00103816 mode $=$ alléteca $=$ GeoWeb +-+ Marciana $)$.

un riflesso nelle descrizioni e nelle elaborazioni grafiche dei secoli successivi, dalle quali possiamo intuire che la cittadella veneziana non doveva apparire troppo diversa, vista da fuori, da quella ottomana in esse ritratta (fig. 4). Al suo interno, oltreal palazzo del bailo, tra gli edifici più rappresentativi dovevano spiccare le due chiese di Santa Maria e San Marco (fig. 5), quest'ultima adiacente ad una piazza, o crocevia, dotata di una loggia ${ }^{23}$. In ottemperanza agli obblighi derivanti dalla sua dipendenza dal monastero di San Giorgio Maggiore di Venezia, il priore della chiesa, al momento di prendere servizio, era tenuto a firmare una promissione, e siamo abbastanza fortunati da poter leggere uno di questi documenti, datato 1270, che contiene un elenco di tutte le suppellettili appartenenti a questo edificio. Secondo tale carta, il priore Benedetto si prenderà cura per cinque anni di tutti i beni della chiesa di San Marco a Negroponte, sia immobili che mobili, ovvero di tutto ciò che serve all'officiatura quotidiana del monastero, obbligandosi a non alienarli né manometterne in alcun modo l'integrità. Segue un elenco dettagliato, che include un gran numero di oggetti ${ }^{24}$. Il testo integrale di questo documento

\footnotetext{
${ }^{18}$ Come ricordato da A. LAIOU, op. cit. (n. 4), p. 17-18, negli statuti della gilda dei pittori si introdurranno provvigioni particolari per oggetti decorati che venivano realizzati specificamente per il Levante.

${ }^{19}$ A. BALLIAN, Liturgical Implements, in H. C. Evans (ed.), Byzantium: Faith and Power. 1261-1557. Catalogue of the exhibition held at The Metropolitan Museum of Art, New York, from March 23 through July 4, 2004, New York, 2004, p. 117-124.

${ }^{20}$ A. KÜLZER, Ostthrakien (Europa), Wien 2008 (Tabula Imperii Byzantini, 12), p. 607-613.

${ }^{21}$ Un recentissimo convegno ha sviluppato questo tema: Neue Forschungen zur spätbyzantinischen Goldschmiedekunst (13.-15. Jh.), Mainz, RömischGermanisches Zentralmuseum, 29-30/10/2015. A mo' di riferimento, con trattazione di diversi casi specifici, si veda P. HETHERINGTON, Enamels, Crowns, Relics and Icons. Studies on Luxury Arts in Byzantium, Aldershot, 2008 (Variorum Collected Studies Series, 908).

${ }^{22}$ Per la storia di Negroponte (attuale Eubea) durante il periodo bizantino e, più in particolare, quello della dominazione veneziana dell'isola, con cenni sia storici che di carattere archeologico, si veda principalmente J. KODER, Negroponte. Untersuchungen zur Topographie und Siedlungsgeschichte der Insel Euboea wärend der Zeit der Venezianerherrschaft, Wien, 1973 (Veröffentlichungen der Kommission für die Tabula Imperii Byzantini, 1); più recentemente N. D. KONTOGIANNIS, Euripos - Negroponte - Eğriboz: Material Culture and Historical Topography of Chalkis from Byzantium to the End of the Ottoman Rule, in Jahrbuch der österreichischen Byzantinistik, 62, 2012, p. $29-56$.

${ }^{23}$ J. KODER, op. cit. (n. 22), p. 90-92. Specificatamente sulla chiesa di San Marco si vedano: M. POZZA, Le chiese veneziane intitolate a San Marco nell'Oriente mediterraneo nei secoli XII e XIII, in A. Niero (ed.), San Marco: aspetti storici e agiografici. Atti del Convegno internazionale di studi, Venezia 26-29 aprile 1994, Venezia, 1996, p. 611-626, in part. p. 614-615; S. BORSARI, La chiesa di San Marco a Negroponte, in Medio Evo Greco, o, 2000, p. 27-34. D. JACOBY, La consolidation de la domination de Venise dans la ville de Négrepont (1205-1390): un aspect de sa politique coloniale, in C. Maltezou, P. Schreiner (eds.), Bisanzio, Venezia e il mondo franco-greco (XIII-XV secolo). Atti del Colloquio internazionale, Venezia 1-2 dicembre 200o, Venezia 2002 (rist. in ID., Latins, Greeks and Muslims: Encounters in the Eastern Mediterranean. $10^{\text {th }}-15^{\text {th }}$ Centuries, Farnham-Burlington, 2009, nr. IX).

${ }^{24}$ ASVe, San Giorgio Maggiore, b. 126, n. 38: « [...] Bonis vero usualibus dictae ecclesiae quae sunt: Crux una argentea, Calix argenteus unus, Turribulum argenteum unum, Panni pro altare duo, Culcita una, Pannum unum et linteamina duo pro mortuis, Paramentum unum cum Casubla una alba et unum aliud Paramentum sine Casubla, Cappa una. Toalie due de altare et alii Panni qui sunt cottidie in altare, Missalle unum, Breviarium unum divisum in duobus volu-
} 


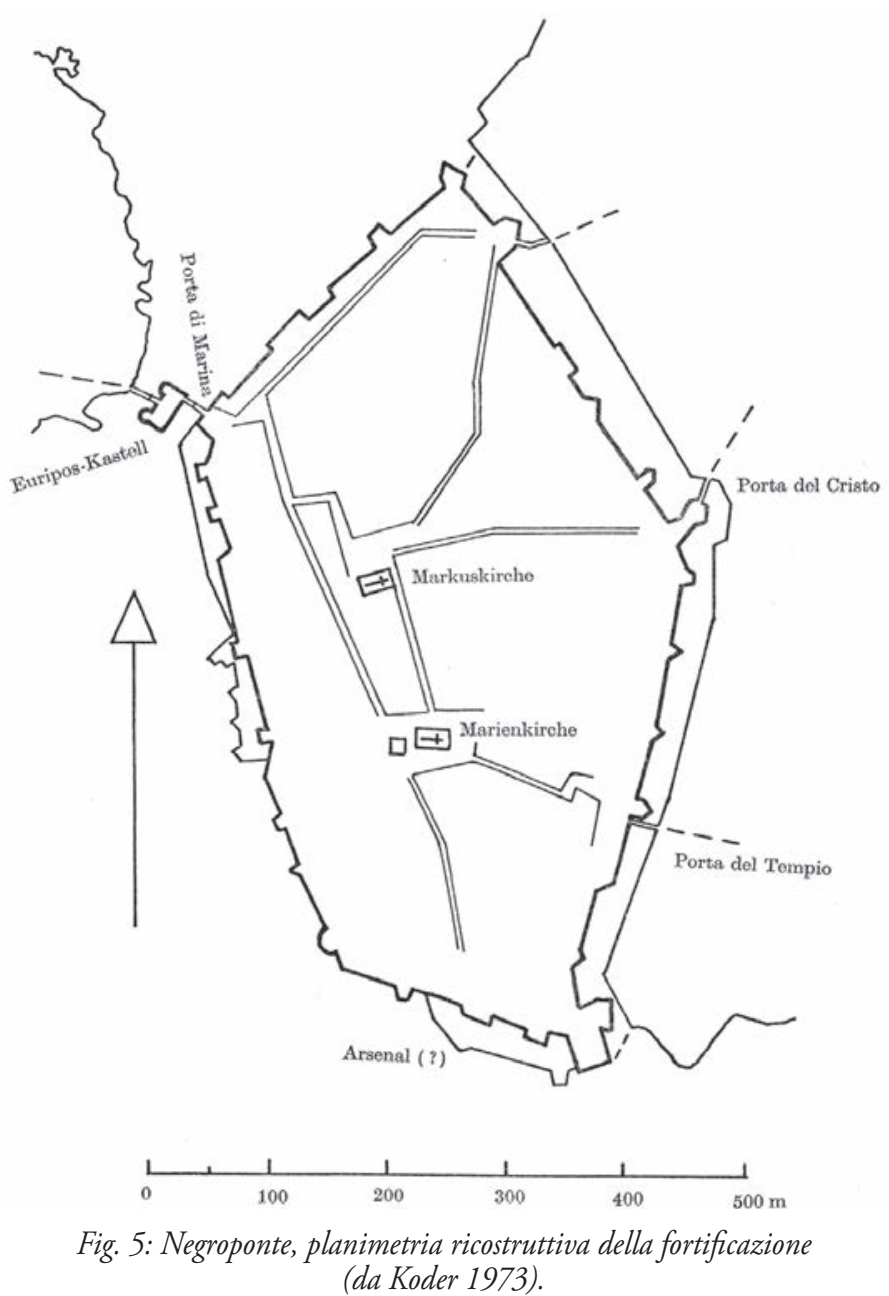

che, a quanto ci risulta, è inedito, presenta ampie lacune, dovute al cattivo stato di conservazione della pergamena lungo tutto il lato sinistro, ma un riscontro a tale elenco si trova in un altro documento del 1274 (anch'esso edito solo a livello di regesto) ${ }^{25}$. Il primo posto è occupato dalle argenterie, che comprendono: una croce (non si specifica se si tratti di una croce processionale o di una croce d'altare ${ }^{26}$ ), un calice e un incensiere (turribulum) anch'esso argenteo. Più numerosi i tessuti, dei quali si indica anche la funzione: panni per l'altare tra cui due tovaglie e altri panni che stanno sull'altare ogni giorno (intendendo quindi differenziare i teli esposti sulla mensa eucaristica durante le celebrazioni da quelli lasciati a copertura dell'altare al di fuori di esse); un cuscino (culci$t a$ ), un panno e due tessuti di lino per i morti (pro mortuis); inoltre, sono inclusi nell'elenco vari paramenti con o senza casula abbinata, una cappa e una dalmatica, e una tunicella di sciamito verde. Si enumerano poi nel dettaglio i libri liturgici in dotazione alla chiesa (messali, manuali, salteri, passionari, sequenziari, istoriali, epistolari ed evangelistari), dei quali si indica anche il numero dei volumi, laddove necessario ("un breviario diviso in due volumi", "un breviario e un messale riuniti in un unico volume"), nonché alcune specifiche che fanno riferimento alla pratica liturgica ("due antifonari per la notte e tre per il giorno"), e in un caso persino al tipo di scrittura con cui i testi sono vergati: «Istoriale unum de littera breventana (sic) », cioè "un istoriale in scrittura beneventana" ${ }^{27}$. Quest'ultima indicazione si direbbe essere una conferma indiretta della provenienza dell'oggetto - in questo caso, a differenza dei sacri vasi - dall'Occidente.

La chiesa di San Marco di Negroponte non esiste più, essendo stata sostituita dopo il 1470 con una moschea ${ }^{28}$, in seguito alla conquista turca. Alcuni anni prima, quando l'isola era ancora nelle mani di Venezia, il cappellano del reggimento di Negroponte, Paolo Pasqualigo, scriveva all'abate di San Giorgio Maggiore, descrivendo la sua indefessa attività di recupero di opere preziose di proprietà della chiesa ${ }^{29}$. La lunga lettera che egli invia al suo superiore è datata 1454. Con questo singolare testo, scritto ormai in veneziano, Pasqualigo aggiorna diligentemente l'abate sullo stato delle proprietà immobili affittate del monastero, ma, soprattutto, il cappellano mostra di essere davvero molto attento al decoro della chiesa anche sul piano artistico: ha infatti subito provveduto a commissionare un bel crocifisso: «Quanto alla giexia vostra ho fatto $1^{\circ}$ bellissimo crucifixo con S. Maria e S. Zuane che basteria fina in la giexia de miser S. Marcho ». Egli si mostra assai solerte anche in una difficilissima impresa, quella di ricercare e recuperare oggetti di proprietà del monastero che, si è scoperto, erano stati alienati o impegnati: alcuni di essi sono già stati recuperati, per altri si farà subito un tentativo a tal scopo, ma è necessario che l'abate faccia pervenire al più presto una procura ufficiale, anche se la legge è senz'altro favorevole al cappellano e coerente con le sue decisioni: egli afferma infatti che vi è « una leze in mio favor che dize che chosse de giexia non se puol impegnar. ..., volio far far $1^{a}$ scumenegazion, azo i possa invegnir in quelle cosse che son state usurpade ». Egli ha già rintracciato un discreto numero di oggetti, per i quali fornisce un elenco con osservazioni sul loro stato di conservazione, offrendo persino qualche raro elemento descrittivo: una croce coperta d'argento; un'icona della Vergine anch'essa parzialmente rivestita d'argento; un

minibus, Antiphonaria de nocte duo, et tres de die, Manuale unum, Psalteria tria, Passionarium unum, Sequentionarium unum, Breviarium unum et Missalle unum ambo in uno volumine, Epistularium et Evangelistarium, Istoriale unum de littera breventana, fora una de casubla una de panno, Dalmatica una et tunicella una de examito viride [...] » (1270, 10 gennaio, indizione XIII, Venezia). La trascrizione è a cura di chi scrive. Cfr. il regesto in E. ORLANDO, op.

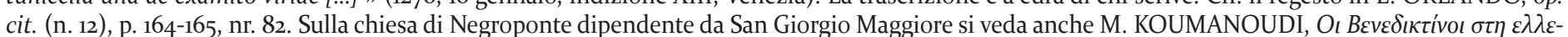

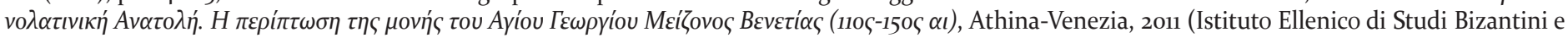
Postbizantini, Venezia. Thomas Flanginis, 6).

${ }^{25}$ ASVe, San Giorgio Maggiore, b. 126, n. 41. Marco Bollani, abate di San Giorgio Maggiore, fa commissione a Benedetto, priore di San Marco di Negroponte di esigere un pagamento da Giovanni da Bologna per la chiesa di San Giorgio a Tebe e di occuparsi di tutti gli affari riguardanti San Marco a Negroponte. Cfr. E. ORLANDO, op. cit. (n. 12), p. 165, nr. 83; Orlando fa presente che del testo di questo documento sarebbe fornita un'edizione in E. GARLATO, San Giorgio Maggiore e il Levante nel XIII secolo, Tesi di Laurea, Università "Ca' Foscari" di Venezia, Facoltà di Lettere e Filosofia, Corso di Laurea in Storia, relatore G. Ortalli, a.a. 2001-2002. Quest'ultima opera è stata, finora, a me inaccessibile.

${ }^{26}$ M. COLLARETA, op. cit. (n. 16), p. 313-315, e P. J. GEARY, op. cit. (n. 16).

${ }_{27}$ "Breventana" può ritenersi corrispondente a "beneventana", termine quest'ultimo già in uso almeno dall'XI secolo per identificare la caratteristica scrittura di origine italomeridionale (ma diffusa anche in area dalmata). Cfr. A. E. LOWE, The Beneventan Script. A History of the South Italian Minuscule, Oxford, 1914 ( ${ }^{\text {nd }}$ ed. prepared and enlarged by V. Brown, Rome 1980). Ringrazio la Dott.ssa Giulia Perucchi (Università di Messina) per la cortese segnalazione.

${ }^{28}$ J. KODER, op. cit. (n. 22); N. D. KONTOGIANNIS, op. cit. (n. 22).

${ }^{29}$ ASVe, San Giorgio Maggiore, b. 126, s.n.. Cfr. E. ORLANDO, op. cit. (n. 12), p. 113-114, 188, nr. 132; M. KOUMANOUDI, op. cit. (n. 24), p. 146-147. 
turibolo d'argento dorato mancante del piede; una piccola navicella da incenso priva del coperchio; una patena d'argento. Il religioso si spenderà per ottenerne la restituzione quanto prima. Tra gli oggetti che è riuscito a recuperare, invece, vi sono: un messale, che era stato impegnato; alcuni libri; vari paramenti, alcuni dei quali egli ha fatto riparare per poterli rendere nuovamente utilizzabili. Egli ha provveduto poi all'acquisto di suppellettile nuova, tra cui delle tovaglie, e ha ricevuto dall'arcivescovo locale un calice del valore di 8 once. Tuttavia ritiene che parte di questi argenti e libri non siano più necessari a Negroponte, e propone di inviarli a Venezia. Pasqualigo si dilunga a riferire tutte le migliorie apportate all'arredo della chiesa, la sua attenzione a far sì che l'illuminazione vi sia sempre adeguata, il restauro urgente del solaio e delle finestre della propria abitazione. Per giunta, ha portato a termine tutto ciò a proprie spese, lamentandosi del fatto che le offerte siano scarse: « non vedo alchun che metesse $1^{\circ}$ soldo per adornar la vostra giexia »- e dunque, in questo modo "battendo cassa" presso il proprio superiore, per sollecitare l'invio del denaro occorrente ${ }^{30}$.

Il documento finisce così per fornire un colorito spaccato dell'attività quotidiana di un chierico, investito di responsabilità amministrative nella colonia e desideroso di mostrarsi all'altezza del compito. L'interesse principale di questa fonte per noi, a parte il raro dettaglio con cui vengono elencate le opere suntuarie, risiede soprattutto nel fatto che proprio una delle ultime testimonianze della presenza veneziana sull'isola - in un momento cruciale di passaggio - è quella dell'attività di raccolta $\mathrm{e}$ - per usare un termine moderno - rudimentale "tutela" di opere d'arte, perseguita certo per motivi pratici, in un contesto storico complesso, fitto di turbolenti mutamenti politici e culturali, nel quale si sarebbe potuto pensare che vi fosse un controllo meno accurato da parte delle autorità oltremare.

\section{VENEZIA, A MO' DI CONCLUSIONE}

Come si è visto, non solo gli oggetti preziosi (per loro natura soggetti a dispersione), ma anche gli edifici, di cui si è fatta menzione, sono andati irrimediabilmente perduti, rendendo ancora più complessa ogni analisi che riguardi la loro storia artistica. Per di più, allo stato attuale degli studi, quasi nessuna opera sopravvissuta può essere ricondotta con ragionevole certezza al milieu dei quartieri veneziani d'Oltremare. Tra i pochissimi esempi in tal senso si può menzionare la celebre icona a micromosaico raffigurante le Dodici Feste, ora a Firenze, donata al Battistero fiorentino,

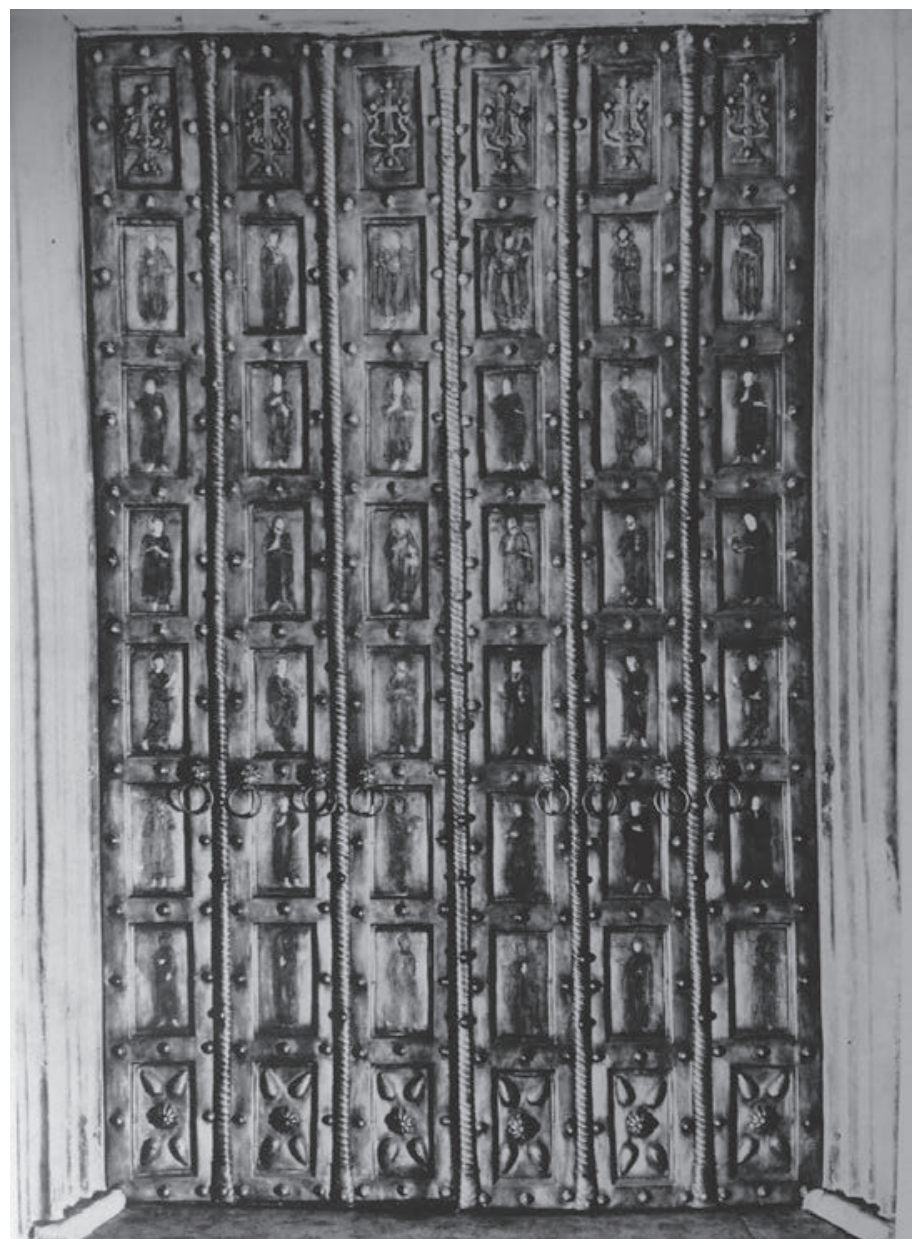

Fig. 6: Venezia, San Marco, Porta bronzea dellingresso centrale (da Forlati 1969).

alla fine del Trecento, da una veneziana residente a Costantinopoli di nome Nicoletta Grioni. Ma su questa complicata e a tratti misteriosa vicenda mi riprometto di soffermarmi in una prossima occasione ${ }^{31}$.

Se i nomi di Veneziani a Bisanzio implicati nella circolazione di beni "di lusso" abbondano nei documenti, lungo tutto il periodo medievale, più difficile è dare un volto ai protagonisti di questa epopea. Tuttavia in un caso ciò è possibile: dobbiamo però tornare indietro, al torno di anni da cui siamo partiti, ovvero al passaggio tra l'XI e il XII secolo, momento in cui si colloca, lo ricordiamo, la donazione della chiesa di Sant'Akindynos, per così dire, dallo Stato alla Chiesa. Leo da Molino - personaggio il cui profilo ci sfugge, forse un

\footnotetext{
${ }^{30}$ Riportiamo qui alcuni passaggi della lettera (la trascrizione è a cura di chi scrive): « Quanto alla giexia vostra ho fatto $1^{\circ}$ bellissimo crucifixo con S. Maria e S. Zuane che basteria fina in la giexia de miser S. Marcho. E cussi andero regulando la vostra giexia, quanto a me sera possibille pur a vostro avixo, spendo del mio [...] i son certo ne siate contento, ma voria aver qualche aiutorio, queste de qui non son ati alimoxina, le casse vostre vano chome le vano, e non vedo alchun che metesse $1^{\circ}$ soldo per adornar la vostra giexia [...]. Se avesse vostra procura et avesse le vostre carte over instrumenti [...] vi prometo de redrezar molte cosse, niente de men faza e dispona la vostra paternità quello ipiazi. E ho tanto cerchado, che ho trovado algune vostre cosse in man de piuxor persone et ancor spiero de recuperar benche non abia vostra procura ma per eser cosse che aspeta alla giexia la raxon me sovien.

E pria $1^{a}$ croxe choverta darzento, post anchona $1^{a}$ de nostra dona con $1^{\circ}$ pocho darzento; item $1^{\circ}$ teribullo darzento dorado senza el pe; item $1^{a}$ navexella pizolla senza sua coverta. Ancor ho saputo eser in man de $1^{\circ}$ prete $1^{a}$ patena darzento, e fin a qui non ho la dicta recuperada, ma più presto i potro, farò de averla. Item ho recuperado $1^{\circ}$ mesal era in pegno, e per eser una leze in mio favor che dize che chosse de giexia non se puol impegnar, hollo avuto, volio far far $1^{a}$ scumenegazion, azo i possa invegnir in quelle cosse che son state usurpade. Ne son alguni libri i qual son mezi alguni paramenti che non se puol adoperar, era $1^{\circ}$ paramento et hollo conzado, con el qual cellebro. Tovaie non era in dita giexia pur fizi con isignor consieri el baillo non siando de opinion che iano comprato mantelli 3 per laltar. [...] Miser larzivesco ma dato $1^{\circ}$ challexe de oz. 8 e lui atolto in si certi arzenti afato stimar el challexe e i arzenti a vostro avixo, credo abi fatto bene e basti. Siche, reverende pater, questi arzenti e libri che me atrovo, me pareria i dovesse mandar alla vostra paternità, perché de qui non son necessarii commanda la vostra paternita quello ipiaxe.

[...] E perché regnum meum non est [...] Lasserò giexia e tute vostre cosse si ben in ordene, che da Dio ne sero premiado e dal mondo saro lauda [...]. » (1454, 19 maggio, Negroponte).

${ }^{31}$ L. BEVILACQUA, Veneziani a Costantinopoli. Qualche considerazione sulla circolazione di opere d'arte nel Mediterraneo nel XIV secolo, comunicazione presentata all'VIII Congresso Nazionale dell'Associazione Italiana di Studi Bizantini, Ravenna, 22-25 settembre 2015.
} 


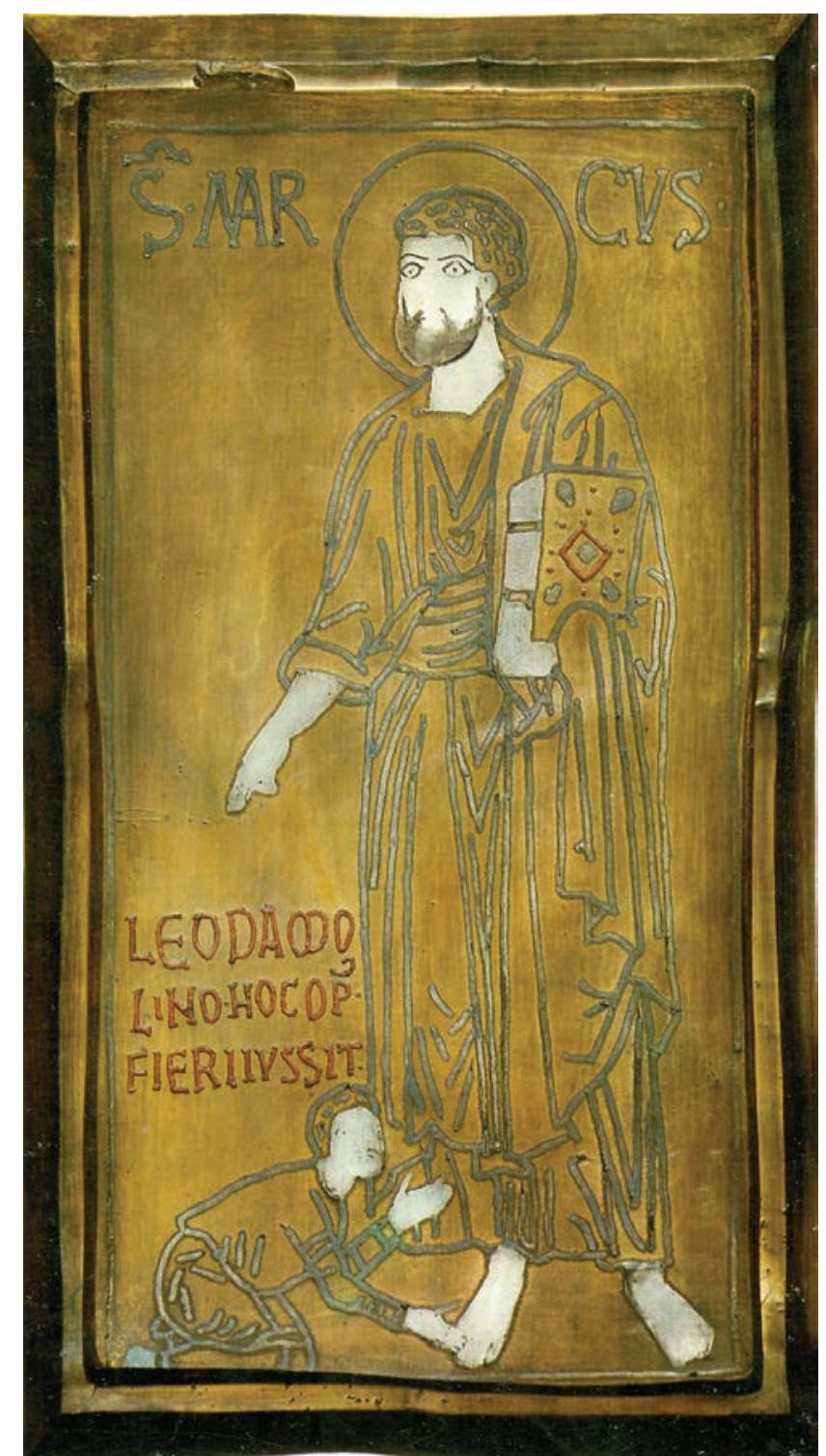

Fig. 7: Venezia, San Marco, Porta bronzea dell'ingresso centrale, particolare: ritratto del committente (da Forlati 1969).

mercante veneziano attivo a Costantinopoli a cavallo tra XI e XII secolo - ha fatto ageminare il proprio ritratto sulla porta centrale della basilica di San Marco (fig. 6), che fu posta in opera proprio agli inizi del XII secolo. Non mi soffermo su questi battenti, che sono stati oggetto di studi approfonditi da parte di specialisti già in passato ${ }^{32}$, ma mi sembra che la figura di Leo possa essere di notevole interesse. Anche se le informazioni su di lui sono estremamente scarse ed incerte, sappiamo che fu un illustre rappresentante della repubblica di Venezia, se è da identificare con questo personaggio colui che nel 1112 sarà insignito della carica di procurator de rispetto. Ma c'è di più, giacché possiamo ragionevolmente presumere che i suoi rapporti d'affari si svolgessero proprio sulle rive del Corno d'Oro. In vari documenti dell'Archivio di Stato di Venezia, infatti, già nell'XI secolo, in più occasioni sono nominati membri della famiglia da Molin (una famiglia certo assai ramificata) quali sottoscrittori in qualità di testimoni di documenti rogati in Oriente, principalmente a Costantinopoli, ma anche ad Almiro e Acri33 ${ }^{33}$. Si tratta solo di un indizio ma, credo, importante, perché dimostra l'effettiva esistenza di contatti diretti tra la famiglia di Leo e l'ambiente costantinopolitano. Sul Corno d'Oro, dunque, egli poté far realizzare un prezioso arredo da donare alla chiesa ducale della sua città d'origine, edificio che si avviava a diventare il più importante per concentrazione di capolavori artistici nella laguna. Egli scelse di apporvi il proprio sigillo di committente, facendosi rappresentare su una delle formelle, in proskynesis di fronte a San Marco (fig. 7). La figura storica di Leo da Molino è ancora da indagare, e ci si chiede quali potessero essere i suoi rapporti, da un lato, con la capitale bizantina, dall'altro quelli intrecciati in patria, con il doge e il suo seguito. La domanda è destinata, per il momento, a rimanere aperta.

Abbiamo qui offerto una panoramica molto vasta del problema, di cui sono stati selezionati solo alcuni "campioni”, e dai cui percorsi sono rimaste escluse per ora altre zone, ad esempio, i centri della Morea, Creta, Cipro, e le rive più orientali e quelle meridionali del Mediterraneo. Si tratta evidentemente di una rete di testimonianze che ha ancora maglie molto larghe, fatte forse solo di indizi, di piccoli tasselli, ma tuttavia utile, ci auguriamo, a una migliore comprensione di un contesto ancora sfuggente e, in ultima analisi, a connettere un sempre maggior numero di documenti e opere al loro contesto di provenienza, anche laddove esse non rechino impressa la loro griffe d'origine.

\footnotetext{
$\overline{3^{2}}$ Per ricordare solo i principali e i più recenti: B. e F. FORLATI, Le porte bizantine di San Marco, Venezia 1969; M. ENGLISH FRAZER, Church Doors and the Gates of Paradise: Byzantine Bronze Doors in Italy, in Dumbarton Oaks Papers, 27, 1973, p. 145-162; A. PARIBENI, Le porte ageminate della basilica di S. Marco a Venezia tra storia e committenza, in A. Iacobini (ed.), Le porte del Paradiso. Arte e tecnologia bizantina tra Italia e Mediterraneo. XI-XII secolo, Roma, 2009 (Milion. Studi e ricerche d'arte bizantina, 7), p. 301-317; E. VIO, Le porte bizantine di Venezia: storia e restauro, Ibid., p. 283-300; R. POLACCO, Le due porte ageminate della basilica di S. Marco a Venezia, in G. Camelia, G. Cobalto (eds.), Fieri iussit pro redemptione. Mecenatismo, devozione e multuculturalità nel Medioevo amalfitano, Amalfi, 2009 (Biblioteca amalfitana, 13), p. 295-297.

${ }_{33}$ Ad esempio in numerosi esemplari conservati nel fondo San Giorgio Maggiore, ma anche, in gran numero, nell'Archivio della famiglia da Molin in San Zaccaria (Luigi Lanfranchi [ed.], Codice Diplomatico Veneziano (secc. XI-XIII), Venezia, 1940-1984). Devo la segnalazione di alcuni di questi documenti alla Prof.ssa Elisabetta Molteni, che desidero qui ringraziare.
} 